\title{
Deuils d'enfance. Sarah Kofman, Rue Ordener, rue Labat
}

\author{
Anne Martine Parent
}

This article proposes a reading of the French philosopher Sarah Kofman's last book, Rue Ordener, rue Labat, published shortly before her death in 1994. Her first and only narrative after several philosophical essays (on Nietzsche and Freud among others), Rue Ordener, rue Labat is about Kofman's childhood in Paris during World War II. The article addresses the death of her father in Auschwitz, and the troubled relationship to her mother.

En 1994, quelques mois avant son suicide, Sarah Kofman fait paraitre un court récit autobiographique: Rue Ordener, rue Labat. Le texte revient sur l'enfance de Kofman et surtout sur les années de l'Occupation, de la rafle du 16 juillet 1942 dans laquelle son père fut pris jusqu'à la fin de la guerre. Le silence de 50 ans entre les événements et leur écriture est révélateur des déchirures qui ont profondément marqué non seulement l'enfance de Kofman, mais également son destin intellectuel.

\section{Le stylo}

La mort du père dans un camp de concentration, déjà évoquée dans Paroles suffoquées (1987), est au fondement du récit. Dès les premiers mots, le père est présent, de manière elliptique, comme un référent absolu qu'il n'est pas nécessaire de nommer: "De lui, il me reste seulement le stylo » (9). Présent par sa disparition et par la rareté des objets qui rappellent son existence: à part le stylo, Kofman parlera également d'une photo du père datant d'avant son mariage, « une vieille photo marron tout abîmée » (63-64) qui la bouleverse énormément, car la jeunesse du père sur cette photo tranche cruellement, aux yeux de celle qui la regarde, avec le destin qui se profile devant lui (« il ne sait pas ce qui l'attend », 64). Un autre objet est également mentionné, qui signe l'effacement du père de manière exemplaire: une carte, envoyée de Drancy, écrite de la main d'un autre en français, langue que le père n'utilisait pas avec sa famille. Carte de lui, donc, mais écrite par un autre dans une langue étrangère et qui, de plus, a disparu: métonymie de la violence faite au père dont les derniers mots à sa famille sont ceux d'un autre et qui sera enterré vivant à Auschwitz, à coups de pioche, effacé avant même d'être mort. La perte de la carte, dernier signe du père, signe de sa disparition, constitue une deuxième mort se surajoutant à la première: "C'était comme si j'avais perdu mon père une seconde fois. Rien ne restait plus désormais, même plus cette seule carte qui n'avait pas été écrite de sa main » (16), ni dans sa langue. 
Rien ne reste plus désormais que le stylo dont Kofman se servira durant toute sa scolarité, ce qui n'est pas sans importance lorsqu'on pense à la manière dont la mère va s'opposer aux études de sa fille. Kofman a dû lutter quotidiennement pour pouvoir continuer le lycée et faire ses devoirs et elle devait souvent lire sous les draps avec une lampe électrique. Dans ce contexte, le stylo devient 1'appui symbolique du père dans la poursuite de la voie intellectuelle choisie par la fille. D'autant plus symbolique que, devenue philosophe, la fille écrira de nombreux livres.

Mais le stylo n'accompagnera pas Kofman durant toute sa carrière, pas " activement 》 du moins: il la « lâche » avant qu'elle-même ne se décide à l'abandonner. C'est donc elle qui est « abandonnée » par lui, comme elle a été abandonnée de son père le 16 juillet 1942 :

En lisant la première fois dans une tragédie grecque les lamentations bien connues "ô popoï, popoï, popoï" je ne puis m'empêcher de penser à cette scène de mon enfance où six enfants, abandonnés de leur père, purent seulement crier en suffoquant, et avec la certitude qu'ils ne le reverraient jamais plus: "ô papa, papa, papa". (14, je souligne).

Dans ce passage où se côtoient la littérature, qui sera un des objets d'étude de Kofman, et la scène d'enfance traumatique, les lecteurs de Kofman auront reconnu une variation du titre d'un de ses livres: Paroles suffoquées (1987). La référence à ce livre n'est pas innocente: livre-hommage à Maurice Blanchot et à ses écrits sur la Shoah dans lequel Kofman se consacre à une lecture du témoignage concentrationnaire de Robert Antelme (L'espèce humaine), l'ouvrage est également écrit en mémoire du père. C'est d'ailleurs dans ce livre qu'on trouvera, raconté pour la première fois, le récit de la mort de Berek Kofman (4142). Dans Paroles suffoquées s'entremêlent donc étroitement le travail intellectuel et le trauma de la mort du père; posture énonciative complexe que revendique explicitement Kofman d'entrée de jeu: « [...] s'impose à moi, intellectuelle juive qui ai survécu à l'holocauste, de rendre hommage à Blanchot [...] »(13-14); « Parce qu'il était juif, mon père est mort à Auschwitz: comment ne pas le dire ? [...] comment ne pas en parler, alors que le vœu de tous ceux qui sont revenus et il n'est pas revenu — a été de raconter [...] » (15-16).

Si dans Paroles suffoquées la réflexion intellectuelle apparaît comme intimement liée à la mort du père, Rue Ordener, rue Labat nous apprend qu'en fait, l'événement de la mort du père, que Sarah Kofman désigne comme son " absolu », constitue le fondement même des travaux philosophiques de Kofman, le moteur de son parcours intellectuel. Cet événement absolu ne pourrait avoir de meilleur représentant que le stylo, le seul objet qui reste, « un stylo comme l'on n'en fait plus » (9), dernier vestige d'un passé disparu mais toujours présent et qui non seulement motive l'écriture, mais la force: 
Je le possède toujours, rafistolé avec du scotch, il est devant mes yeux sur ma table de travail et il me contraint à écrire, écrire... Mes nombreux livres ont peut-être été des voies de traverse obligées pour raconter "ça". $(1994,9)$

\section{Rue Ordener, rue Labat}

Curieusement, alors que le premier chapitre du livre qui parle du stylo du père pourrait laisser croire au lecteur que la mort du père sera l'événement central du livre, son "sujet », cette histoire est relativement rapidement évacuée et laisse place à une autre histoire, liée à la mère. L'histoire du père n'occupe que cinq chapitres sur vingt-trois; puis rapidement, le récit se concentre sur la mère et ses enfants, qui doivent se débrouiller avec les rafles de plus en plus nombreuses. La mère place ses six enfants à la campagne, mais la petite Sarah refuse de manger (en particulier la viande de porc); ce prétexte d'obéissance à la loi paternelle lui sert, inconsciemment ou non, à retourner auprès de sa mère. " Le vrai danger: être séparée de ma mère » (33).

Ainsi, derrière l'événement « absolu » qui marque l'enfance de Sarah Kofman, se cache un autre drame: la séparation d'avec la mère. Drame en deux temps puisque la mère, d'abord aimée, deviendra une figure ennemie. Le titre du livre renvoie au conflit qui structure le récit, conflit qui se joue sur deux « espaces ». Dans la rue Ordener se trouve l'appartement de la famille Kofman, le lieu de la famille et du judaïsme (le père de Sarah est rabbin et la vie de la famille est rythmée par les fêtes et les interdits religieux). Lieu du danger aussi, car on viendra y chercher le père et, plus tard, la mère et les enfants, cette fois sans succès puisque, prévenue de la rafle, la mère, qui avait déjà « placé » cinq de ses enfants à la campagne, est allée se réfugier chez « la dame de la rue Labat » avec Sarah, qu'elle avait finalement gardée auprès d'elle. La dame de la rue Labat, que Sarah appelle mémé, est une ancienne voisine des Kofman qui aimait beaucoup les enfants. Se souvenant de cet amour pour les enfants, la mère de Sarah décide de chercher refuge chez elle. Ce qui devait durer une seule nuit s'est finalement prolongé jusqu'à la Libération, puisque l'appartement des Kofman a été saccagé et mis sous scellé, et que toute la famille est recherchée. La rue Labat constitue donc le lieu-refuge, mais le départ de la rue Ordener sera pour Sarah l'occasion d'une rupture d'avec la mère et le judaïsme.

Cette séparation que réussit mémé, «à son insu ou non », représente un véritable « tour de force » (57), puisque jusque-là, Sarah avait absolument refusé de s'éloigner de sa mère, au risque de sa vie. Par mesure de sécurité, la mère tente à plusieurs reprises de la cacher dans des familles non-juives, à la campagne ou à Paris (où elle peut plus souvent la visiter) et même dans un hôpital et chez des curés. À chaque fois, Sarah refuse de manger et sa mère doit la reprendre. L'angoisse de séparation d'avec la mère trouve un moyen d'expression privilégié dans la nourriture: refus de s'alimenter et, surtout, de ne pas manger kasher. L'attachement à la mère et le respect des interdits religieux sont ici inextricablement liés de la même manière que, plus tard, le détachement de la mère s'accompagnera de l'abandon des pratiques religieuses ( $j$ 'y reviendrai). 
Ainsi, le rapport à la nourriture marque l'évolution des rapports entre Sarah et sa mère, et Sarah et mémé, la deuxième figure maternelle. Le départ précipité de la rue Ordener pour la rue Labat (sous la menace d'une rafle) est d'ailleurs le lieu d'une scène exemplaire: pour passer d'une rue à l'autre, il faut emprunter la rue Marcadet; celle-ci parait à Sarah « interminable » et elle vomit « tout le long du chemin " (40). Ce rejet de la nourriture maternelle - car Sarah et sa mère mangeait un bouillon de légumes vraisemblablement préparé par celle-ci au moment où elles ont été prévenues de la rafle - tout le long de la rue Marcadet, en route donc vers celle qui deviendra la deuxième mère, préfigure la rupture prochaine d'avec la mère. La rue Marcadet constitue un lieu de transition entre la rue Ordener et la rue Labat, lieu d'une « purge » qui prépare - et rend possible? - le rejet de la mère et l'adoption d'une nouvelle figure maternelle.

C'est également par le biais de la nourriture que se marque le passage d'une mère à l'autre. Dans les premiers temps de l'installation chez la dame de la rue Labat, Sarah continue à manger la nourriture kasher préparée par sa mère qui « sortait à ses risques et périls, pour tenter encore d'en trouver » (48). Mais très rapidement, mémé juge que la nourriture qu'a ingérée Sarah durant toute son enfance est "pernicieuse pour la santé » (48); elle trouve Sarah pâle, " lymphatique ", et décide de prendre en charge son alimentation en la nourrissant entre autres de viande saignante et de porc. Le corps de Sarah refuse d'abord ce nouveau régime, cette intrusion étrangère et forcément inquiétante (51), et la petite fille vomit fréquemment, ce qui met mémé en colère. Cette colonisation du corps de Sarah s'accompagne d'une prise en charge complète de la fillette: nouvelle coiffure, nouveaux vêtements, nouvelles habitudes de vie. «Peu à peu, mémé opéra en moi une véritable transformation » (49); "Elle entreprit de me rééduquer de pied en cap et de parachever mon instruction $»(58)$. Et mémé ne manque pas, au détour, de transmettre quelques préjugés antisémites:

Elle m'apprit que j'avais un nez juif en me faisant palper la petite bosse qui en était le signe. Elle me disait aussi: "La nourriture juive est nocive pour la santé; les Juifs ont crucifié Notre Seigneur JésusChrist; ils sont tous avares et n'aiment que le pognon (sic); ils sont très intelligents, aucun autre peuple ne possède autant de génie en musique et en philosophie". (57)

Parallèlement à l'appropriation du corps de Sarah par mémé, Sarah, de son côté, s'attache aussi de plus en plus à mémé jusqu'à la préférer à sa propre mère. C'est le jour de la fête des Mères qu'elle s'aperçoit que le glissement s'est fait, lorsqu'elle achète deux cartes et choisit de donner celle qu'elle trouve la plus belle à mémé: « J'ai honte et je me sens rougir dans la boutique. Mon choix vient bel et bien d'être fait, ma préférence déclarée » (55); la « fausse » mère a désormais remplacé la « vraie » mère. La fille mange désormais des beefsteaks saignants, ne pense plus du tout à son père et ne peut plus prononcer un seul mot en yiddish (67). Tout se passe comme si le père, une fois disparu, rien ne pouvait plus assurer le maintien de la Loi familiale et religieuse dont il est le gardien - à la fois en tant que père et rabbin. 
Une scène d'un film de Hitchcock, The Lady Vanishes, représente de manière emblématique pour Sarah Kofman le glissement qui s'est opéré d'une mère à l'autre, le remplacement de l'une par l'autre. Kofman parle d'ailleurs de ce film comme l'un de ses films préférés, mais qui fait naitre chez elle une « angoisse viscérale » (75).

La scène anxiogène du film consiste en la disparition de « la bonne petite vieille ", Miss Froy, assise dans le train en face de l'héroïne endormie (prénommée Iris) et en son remplacement par une autre femme, portant les mêmes vêtements. Un pseudo-médecin tente de convaincre Iris qu'un coup qu'elle a eu sur la tête a provoqué des hallucinations et qu'elle a toujours eu devant elle la deuxième fermme, qu'il n'y a jamais eu de Miss Froy (qui est en réalité un agent secret et qui a été enlevée par des espions pendant qu'Iris dormait).

L'intolérable, pour moi, c'est toujours d'apercevoir brutalement à la place du bon visage "maternel" de la vieille (tout dans le film suggère qu'elle est l'image d'une bonne mère [...]), l'intolérable, c'est d'apercevoir brusquement le visage de sa remplaçante (elle a revêtu les vêtements de la bonne vieille [...]); visage effroyablement dur, faux, fuyant, menaçant, en lieu et place de celui si doux et si souriant de la bonne dame au moment même où l'on s'attendait à le retrouver.

Le mauvais sein à la place du bon sein, l'un parfaitement clivé de l'autre, l'un se transformant en 1'autre. (76-77)

Ce passage se donne comme l'interprétation d'un épisode de l'enfance de Sarah Kofman, mais une interprétation qui n'est pas si simple à déchiffrer qu'elle n'y paraît au premier abord. Le mauvais sein à la place du bon sein, c'est mémé, la " fausse " mère - qui fait d'ailleurs passer Sarah pour sa fille pendant l'Occupation lorsqu'elles sortent —, à la place de la « vraie » mère L'angoisse de séparation d'avec la mère s'est déplacée sur mémé; c'est de mémé, dorénavant, que la petite Sarah ne veut pas s'éloigner, faisant des crises et refusant de s'alimenter lorsque sa mère l'empêche de la voir. Mais une lecture attentive de la description de la scène du film de Hitchcock et sa mise en relation avec d'autres passages du récit ouvre d'autres possibilités interprétatives. La vieille Miss Froy au bon visage « maternel » pourrait bien représenter mémé, qui aime les enfants, est affectueuse, prend soin de Sarah, de ses cheveux, ses vêtements, ses études, etc., et lui fait connaître la vie de famille en l'amenant chez sa mère (les Kofman n'ont aucune famille en France), alors que la mère de Sarah est souvent dépeinte comme violente - elle n'hésite pas à frapper sa fille à coups de martinet et Sarah se retrouve souvent couverte de bleus -, dure, et elle fait tout pour empêcher sa fille de poursuivre ses études. De plus, une scène du récit ressemble étrangement au remplacement " brusque » de Miss Froy par la deuxième femme. Après la guerre, la mère de Sarah doit partir pour Nonancourt où se trouvent certains de ses enfants, et elle se voit obligée de confier Sarah à mémé. Les retrouvailles sont "idylliques » (79); Sarah et mémé dorment même ensemble afin de « n'être plus, cette fois, séparées ni de jour ni de nuit » (79). 
Un jour, 4 heures 30 . Fin de l'école. Je me précipite vers la sortie, je cherche mémé. Ce n'est pas elle qui m'attend mais bel et bien ma mère venue me reprendre définitivement. Mon cœur se met à battre à toute allure. Ce fut atroce. Je n'eus même pas le droit d'aller rue Labat dire au revoir. (81)

Le remplacement brutal de mémé par la mère, au moment même où Sarah s'attendait à la retrouver, ressemble fort à la scène de disparition dans The Lady Vanishes (" l'intolérable, c'est d'apercevoir brusquement le visage de sa remplaçante [...], en lieu et place de celui si doux et si souriant de la bonne dame au moment même où l'on s'attendait à le retrouver »). Reste que, encore une fois, ce n'est peut-être pas aussi simple, et la multiplicité interprétative laisse voir qu'il n'y a peut-être pas, après tout, de " bonne " mère. Le drame qui se joue entre Sarah, sa mère et mémé est complexe. Si Sarah préfère effectivement mémé et n'arrive plus à vivre avec sa mère, quand un tribunal décide de la confier à mémé en raison des mauvais traitements que lui inflige sa mère (la petite exhibe les marques causées par le martinet sur son corps), Sarah ressent un malaise devant cette décision et est finalement secrètement soulagée lorsque sa mère vient violemment la reprendre (même si elle fait une crise et en apparence refuse de partir).

Mais la rupture créée par l'intrusion de mémé ne semble jamais se guérir avec les années. Il y a dans le rapport à la mère un affect douloureux qui n'arrive pas à se dire, fait de violence, de déchirements entre les deux mères, de résistance - Sarah refuse souvent de se nourrir pour s'opposer à sa mère - et de honte Sarah a honte de sa mère lorsque celle-ci exhibe sa fierté devant les succès de sa fille, ce qui est révélateur du fossé creusé entre elles, de la désolidarisation causée par l'éloignement. Le récit se termine sur l'évocation de la mort de mémé, mais rien n'est dit sur celle de la mère, comme si la réconciliation était impossible ou la mère était « morte » pour l'enfant pendant la guerre (« tuée » par elle ?), comme le père. Abandonnée par son père, la fille abandonne à son tour sa mère et le récit tente justement de structurer les pertes, les deuils et les déchirures qui ont marqué l'enfance de Sarah Kofman et l'ont constituée comme sujet, afin, peut-être, de l'en délivrer: « L'œuvre d'art », écrit-elle dans L'enfance de l'art (1970), « n'est pas la projection d'un fantasme [mais] en est au contraire un substitut qui permet de le structurer après coup et de s'en délivrer » (127). Cet aspect « structurant » de l'écriture pour Kofman est fondamental; on ne peut lire Rue Ordener, rue Labat comme une simple restitution de souvenirs à laquelle se livre Kofman, une activité d'écriture "légère " par opposition à ses textes philosophiques « sérieux ». Il faut plutôt y voir une réactivation d'affects d'angoisse et de mort, un véritable travail qui vise à réinterpréter les événements de l'histoire psychique d'un sujet afin de saisir l'insaisissable, d'intégrer l'étrangement inquiétant et de tenter de dire ce qui toujours se dérobe. Entreprise qui n'est pas sans danger - et il est difficile, ici, de ne pas songer au suicide de Sarah Kofman quelques mois seulement après la publication du texte - car l'autobiographie, comme l'enseigne Kofman elle-même dans Autobiogriffures 
(1976), est « un tissu toujours rhapsodique, toujours défait par le travail d'un négatif non relevable, par quelque griffure mortifère » (115). Et toujours lacunaire.

\section{Bibliographie}

Kofman, Sarah. L'enfance de l'art. Paris: Galilée, 1970 [1985].

— Autobiogriffures du chat Murr d'Hoffmann. Paris: Galilée, 1976 [1984].

_ Paroles suffoquées. Paris: Galilée, 1987.

_ Rue Ordener, rue Labat. Paris: Galilée, 1994.

Michaud, Ginette. « Résistances du récit (Kofman, Blanchot, Derrida)».

In L'étonnement. Francine Belle-Isle, Simon Harel et Gabriel-Louis Moyal (dir.).

Montréal: Liber, 2000. 191-223. 